\title{
Violence, dissatisfaction and rapid tranquillisation in psychiatric intensive care
}

\author{
Clive E. Hyde, Colina Harrower-Wilson and Julie Morris
}

\begin{abstract}
We examined the aseociations of violence, patient diseatisfaction and occurrence of rapid tranquillisation in psychiatric intensive care, using an on-line nursebased computerised database over a two-year period. Non-Caucasians were over-represented in violent incidents with physical threat, and previous forensic history was associated with more violent means of attack. Dissatisfaction related to non-understandable provocation and the total number of violent incidents. There was no correlation between rapid tranquillisations or side-effects and dissoristaction. Remedial action and education in the psychiatric intensive care unit may reduce violence, and better prescribing hablts, avolding anti-psychotic polypharmacy in rapid tranquillisation, should be encouraged.
\end{abstract}

The aims of the present study were to audit the usage of a psychiatric intensive care unit and the occurrence of violent incidents over a two-year period, and to measure patient dissatisfaction with the service and correlate it with violent incidents, rapid tranquillisation episodes and total side-effects.

\section{The study}

The psychiatric intensive care unit in South Manchester, established in 1992, has 12 beds and serves a population of 175000 (aged 16-65) with 80 other general adult beds on a district general hospital site. Black and ethnic minorities make up $11 \%$ of the catchment population.

To identify and measure violent incidents a nurse-run interactive computer system was established, the Psychiatric Studies in Aggression Database. Daily ratings were made for the first seven days, then weekly, on symptom. general aggression and side-effect rating scales. Specific violent incidents are recorded using the Staff Observation of Aggression Scale (SOAS) of Palmstierna \& Wistedt (1987). A patient/user dissatisfaction questionnaire was included for use on discharge or transfer back to the base ward. It had three groups of scores on dissatisfaction with the ward environment, information made available to people on admission and nurse/doctor contacts.

Rapid tranquillisation events were also recorded through the SOAS and patients' sideeffects during their stay were rated on a simple seven-item three-point rating scale. Items comprised tremor, rigidity, dystonic reaction, akathisia, visual difficulties, tardive dyskinesia and 'other' ratings. All side-effect ratings were totalled over the duration of stay. Rapid tranquillisation was mainly by intramuscular zuclopenthixol acetate or haloperidol, often with the addition of lorazepam intramuscularly. The medication used was down to individual consultant team choice with no overall unit policy at this time. Doses used generally followed British National Formulary guidelines, although higher doses were occasionally used in very disturbed or resistant cases.

\section{Findings}

Two hundred and fifty-one patients were admitted between September 1992 and November 1994. The mean age was 34.6 years, range 16-71 years. One hundred and fifty-seven admissions (62\%) were male and $94(38 \%)$ female. The median length of stay was nine days with a range of $0-131$. Thirty-eight $(15 \%)$ had a previous forensic history. In terms of ethnic grouping. $212(85 \%)$ patients were Caucasian. $15(6 \%)$ were Afro-Caribbean and $12(5 \%)$ were of Indian sub-continent origins. One hundred and seventy-seven patients $(70 \%)$ were detained under the Mental Health Act 1983.

Primary diagnosis was entered by a screenbased menu from the admitting doctor's diagnosis. The primary diagnosis was schizophrenia $(40 \%)$, severe depression (11\%), mania (11\%), manic depression (mixed/rapid mood cycling) (11\%), substance misuse $(8 \%)$, organic psychosyndrome $(3 \%)$, personality disorders $(3 \%)$, neurosis $(0.4 \%)$ and other diagnoses including learning disability (11\%). On clinical grounds two constructed diagnostic groups were also 
examined: a combined manic/mixed or rapid cycling affective group ( $n=55(22 \%)$ ) because of similar behavioural disturbance; and a schizophrenia/personality disorder/substance misuse combination $(n=131(52 \%))$ due to the frequency of mixed or dual diagnoses in this group. This group provided a common source of differential diagnostic debate in young, disturbed, possibly drug intoxicated, acute psychotic admissions.

\section{Violent incidents and their associations}

Of the 251 patients admitted, 121 (48\%) had at least one violent incident during their stay and there were a total of 391 violent incidents rated through the SOAS scale. Of the 121 patients having violent incidents, 59 (49\%) were associated with a non-understandable provocation on the SOAS (Table 1 and 2). Twenty-two (18\%) had an incident involving self-harm. Patients with violent incidents had significantly longer stays than those who did not (median (interquartile range) 14 (7-26) days versus 6 (2-14) days, $P<0.001)$. Those patients with a nonunderstandable provocation of incidents stayed significantly longer compared with those with no such violent incidents (median (interquartile range) $16(8-30)$ days versus $7(3-16)$ days, $P=0.001)$. A significantly higher proportion of non-Caucasian people $(n=21)$ were involved in verbal and physical threat violent incidents than Caucasian people $(n=100)$. For non-Caucians 21

Table 1. Staff Observation of Aggression Scale rating of violent incidents $(n=121)$

\begin{tabular}{lc}
\hline & $n(\%)$ \\
\hline Verbal threat alone & $24(20)$ \\
Verbal plus physical threat & $97(80)$ \\
Means used: & \\
Hands: & $80(66)$ \\
Feet & $56(46)$ \\
Chair & $10(8)$ \\
Glass & $4(3)$ \\
Knives & $5(4)$ \\
Other' & $22(18)$ \\
\hline
\end{tabular}

1. Cups, ties, cigarettes, keys, knees or head-butts.

Table 2. Staff Observation of Aggression Scale sub-scale scores

\begin{tabular}{llll}
\hline & Median & $\begin{array}{l}\text { Interquartile } \\
\text { range }\end{array}$ & Range \\
\hline Means used & 1.7 & $1-2$ & $0-4$ \\
Aims of Aggression & 2.0 & $1-2$ & $0-4$ \\
Results of Aggression & 1.0 & $0-1$ & $0-4$ \\
Total score & 4.3 & $3-5$ & $0-9$ \\
\hline
\end{tabular}

Mild, 2-5; Moderate, 6-8; Severe, 9 or more.
Table 3. Violent incidents by forensic history

\begin{tabular}{llll}
\hline & $\begin{array}{l}\text { Forensic } \\
\text { history } \\
(n=15)\end{array}$ & $\begin{array}{l}\text { No forensic } \\
\text { history } \\
(n=106)\end{array}$ & $\begin{array}{l}\text { Fisher's } \\
\text { exact } \\
\text { test }\end{array}$ \\
\hline Use of chair & $4(27 \%)$ & $6(6 \%)$ & $P=0.02$ \\
Use of teeth & $4(27 \%)$ & $7(7 \%)$ & $\begin{array}{l}P=0.03 \\
\text { Use of knife }\end{array}$ \\
\hline$(27 \%)$ & $1(1 \%)$ & $P=0.0007$ \\
\hline
\end{tabular}

$(100 \%)$ had verbal and physical threat whereas only $76(76 \%)$ of Caucasian people had such incidents (Fisher's exact test; $P=0.01$ ) (Table 3). The total SOAS score would put the overall violence at the level of mild, however nursing injuries included a number of severe punches, a fractured nose, a severe bite wound and the use of objects such as knives and tools in a minority of assaults during this study period.

\section{Affective disorder, violence and self-harm}

Twenty-two (18\%) of the patients self-harmed. There was a significant association with youth (mean age 30 years) compared with no self-harm incidents (mean age 35 years) $(P=0.006)$, and longer stay (median (interquartile range) 22 (949) days versus $9(3-17)$ days, $P=0.001)$. There was a higher incidence of self-harm among the severe depression and schizophrenia/personality disorder/substance misuse groups $(P=0.03)$.

Seventy-seven patients had a violent incident on the same or next day after admission and there was a significant increase in manic/mixed manic-depressive diagnoses in this group. 25/ $55(46 \%)$ had incidents; severe depressive 6/28 (21\%); schizophrenia/personality disorder/drug misuse 36/131 (28\%); organic and others 9/36 $(25 \%)(P=0.046)$.

\section{Patient dissatisfaction scores}

Patient dissatisfaction scores were obtained on the last day of stay for 170 of the 281 patients $(68 \%)$. The scale consisted of 12 items, including dissatisfaction with rooms, information on admission, availability of nurses, ward rounds, meals, ward booklets, explanations of treatment. welcome to the ward, recreational facilities, answers to questions, preparation for discharge and ward decor and surroundings. It could be rated from being totally satisfied to not at all satisfied on a six-point scale. The maximum score for dissatisfaction was 60 , and the mean score was 25.4 (s.d. 11.5, range 0-60). There was no significant difference in dissatisfaction scores between those who experienced rapid tranquillisation events $(n=76)$ and those who did not $(n=84)$ during their stay, and there was no significant correlation with rated side-effects. 
Table 4. Non-understandable violent incidents and dissatisfaction items ${ }^{1}$

\begin{tabular}{lll}
\hline & $\begin{array}{l}\text { No non- } \\
\text { understandable }\end{array}$ & $\begin{array}{l}\text { At least one non- } \\
\text { understandable } \\
\text { incident }\end{array}$ \\
\hline $\begin{array}{c}\text { Information on } \\
\text { admission }\end{array}$ & $2(1-3)$ & $3(2-4) P=0.001$ \\
$\begin{array}{c}\text { Explanation of } \\
\text { treatment }\end{array}$ & $2(1-3)$ & $3(2-4) P=0.002$ \\
$\begin{array}{c}\text { Recreational } \\
\text { facilities }\end{array}$ & $2(1-3)$ & $3(2-4) P=0.008$ \\
$\begin{array}{c}\text { Preparation for } \\
\text { discharge }\end{array}$ & $2(1-3)$ & $2(2-3) P=0.05$ \\
\hline
\end{tabular}

1. Median (interquartile range) Mann-Whitney U-test.

There were, however, two significant associations; with the occurrence of at least one violent incident of an unprovoked type ( $n=40$, mean dissatisfaction score 29.3) no violent incident of an unprovoked nature $(n=130$, mean score 24.2$)$ $(P=0.013)$. Also there was a borderline significant rank correlation with patient dissatisfaction score and the total number of violent incidents $(P=0.058)$.

Examination of differences between individual items of dissatisfaction for patients with at least one non-understandable violent incident indicated that four items showed significant differences between those with no such violent incidents and those with incidents (see Table 4). Typical comments made were: favourable - "it was a pleasant stay, where the staff let me do my own thing and helped me to relax in the surroundings", "the staff make you feel more homely, especially the female staff"; unfavourable - "all I want to know is when do I get released from this establishment", "the food was the pits, there was never enough sent from the kitchen, the staff seemed embarrassed to give it to the patients"; bizarre - "need more colour, love the sound system, I have met some lovely staff and patient mortals". "I did not read the booklet, decor should be Deep Diana Pink". A similar pattern of significant differences $(P<0.05)$ can be found for information and explanation with at least one violent incident of any type during stay.

\section{Side-effects and rapid tranquillisation}

Overall, 194 of 251 patients reported side-effects during their stay $(77 \%)$. The total score during the stay was a median of 2 (interquartile range 15 ; range 0-48). The percentage zero score was 48 (19\%). There was no significant association with patient dissatisfaction.

Patients who had had no rapid tranquillisation events had significantly lower side-effect scores
( $n=94$, median 1, range $=0-48$, per cent zero score $=36 \%$ ) compared with those who had had rapid tranquillisation; either with haloperidol ( $n=54$, median 2 , interquartile range 1-6, range 0-23, per cent zero score $=18 \%$ ) mixed rapid tranquillisation medication ( $n=14$, median 4 , interquartile range 4-7, range 1-27, per cent zero score $=7 \%)$ or zuclopenthixol acetate $(n=32$, median 4 , interquartile range $2-8$, range $0-27$. per cent zero score $=12 \%)(P<0.0001)$. Within the three groups who received some form of rapid tranquillisation the haloperidol group had significantly lower scores compared with individuals who recetved mixed anti-psychotic medication but not those who only received zuclopenthixol acetate for their rapid tranquillisations $(P<0.05)$.

\section{Comment}

Various factors affecting the risk of violence have been the subject of recent work (Sheehan et al, 1995: Thomas et al, 1995) and suggestions have been made as to what can be done about it (Hyde \& Harrower-Wilson, 1994; Atakan, 1995).

Our study demonstrated the possibility of experienced nursing staff in a psychiatric intensive care unit running a comprehensive database over a two-year period. Problems with reliability were addressed by regular teaching, meetings and videotape work.

Findings of interest included the association of violent incidents, including those with nonunderstandable provocation, with longer stay; manic or mixed states with early violence; selfharm with youth, severe depression and the combined diagnoses, schizophrenia/personality disorder/drug misuse not uncommon in current clinical practice. Non-Caucasians were significantly over-represented in physical threat but this was not specific to any single ethnic group. A previous forensic history was associated with particularly violent means of attack.

Dissatisfaction scores on discharge were available for $68 \%$ of admissions and were significantly related to at least one violent incident of nonunderstandable provocation and the total number of violent incidents. Of particular interest was the significant difference between those with and without incidents concerned with items of information, explanation, recreation and preparation for discharge. This suggests areas of remedial action and education in the psychiatric intensive care unit milieu may reduce the occurrence of violence. Consequently we have recently introduced day-time adult education sessions on the psychiatric intensive care unit to apparent good effect. 
There was no correlation between the occurrence or number of rapid tranquillisation events and patients' general dissatisfaction scores. Side-effects from potent antipsychotic drugs used in rapid tranquillisation incidents did not seem to influence dissatisfaction, although rapid tranquillisation was significantly associated with higher total side-effects over the patient stay. Within those groups who received rapid tranquillisation the polypharmacy group who received a number of different antipsychotics had significantly raised side-effects compared with those receiving monotherapies, either with shortacting haloperidol or intermediate-acting zuclopenthixol acetate alone.

Further research needs to examine the role of ethnic grouping in violence, factors influencing dissatisfaction and remedial and educational aspects of patients' stay on psychiatric intensive care unit. Better prescribing habits avoiding antipsychotic polypharmacy in rapid tranquillisation should also be encouraged.

\section{References}

ATAKAN, Z. (1995) Violence on psychiatric in-patient units: what can be done? Psychiatric Bulletin, 19, 593-596.

HYDE, C. E. \& HARROWER-WILSON, C. (1994) Psychiatric intensive care: principles and problems. Hospital Update, May, 287-295.

PALmSTIERNA, T. \& WistedT, B. (1987) Staff Observation and Aggression Scale, SOAS: Presentation and Evaluation. Acta Psychiatrica Scandinavica, 76, 657-663.

Sheehan, J. D. HARDIE, T. \& WATSON, J. P. (1995) Social deprivation, ethnicity and violent incidents on acute psychiatric wards. Psychiatric Bulletin, 19, 597-599.

Thomas, C. BARTlett, A. \& MezeY, G. C. (1995) The extent and effects of violence among psychiatric in-patients. Psychiatric Bulletin, 19, 600-604.

*Clive E. Hyde, Consultant Psychiatrist, Colina Harrower-Wilson, Sub Unit Nurse Manager and Julie Morris, Senior Medical Statistician, South Manchester University Hospital Trust, Withington Hospital, Nell Lane, West Didsbury, Manchester M20 2LR

*Correspondence

\title{
Benzodiazepine prescribing in a psychiatric hospital
}

\author{
J. Summers and K. W. Brown
}

\begin{abstract}
Using a case note study, this paper presents a longitudinal survey of the effect of psychiatric inpatient care on benzodiazepine prescribing. Standards were proposed to assess the quality of this prescribing. Based on these standards, the study shows inappropriate use of benzodiazepines. Following admission, there was an increase in the number of patients prescribed benzodiazepines and in the number of benzodiazepines prescribed. Of the benzodiazepines withdrawn, most were contrary to the proposed standard. The quality of drug history showed little emphasis being placed on rationalising benzodiazepine prescribing. The issue of how benzodiazepines should be handled during psychiatric admission is discussed.
\end{abstract}

In 1988, the Committee on Safety of Medicines declared that the use of benzodiazepines should be decreased as dependence was becoming a subject of increasing concern (Committee on Safety of Medicines, 1988). Benzodiazepine misuse is a further issue which has raised concern. In 1993, temazepam was the most commonly misused drug in Scotland (Scottish Health Service Common Services Agency, 1993). Furthermore, there is evidence from the Home Office Drugs Inspectorate that "the vast majority of misused benzodiazepines are obtained on prescription" (National Medical Advisory Committee, 1994).

Although anxiolytic benzodiazepine use has decreased to about one-quarter of its use 15 years ago (Royal College of Psychiatrists, 1997). the above concerns remain relevant and emphasise the need for high standards in benzodiazepine prescribing. Psychiatrists should be 